\title{
Echo Behavior in Large Populations of Chemical Oscillators
}

\author{
Tianran Chen, ${ }^{1}$ Mark R. Tinsley, ${ }^{1}$ Edward Ott, ${ }^{2}$ and Kenneth Showalter ${ }^{1}$ \\ ${ }^{1}$ C. Eugene Bennett Department of Chemistry, West Virginia University, \\ Morgantown, West Virginia 26506-6045, USA \\ ${ }^{2}$ Institute for Research in Electronics and Applied Physics, \\ University of Maryland, College Park, Maryland 20742, USA \\ (Received 14 August 2016; revised manuscript received 1 October 2016; published 15 December 2016) \\ Experimental and theoretical studies are reported, for the first time, on the observation and \\ characterization of echo phenomena in oscillatory chemical reactions. Populations of uncoupled and \\ coupled oscillators are globally perturbed. The macroscopic response to this perturbation dies out with \\ time: At some time $\tau$ after the perturbation (where $\tau$ is long enough that the response has died out), the \\ system is again perturbed, and the initial response to this second perturbation again dies out. Echoes can \\ potentially appear as responses that arise at $2 \tau, 3 \tau, \ldots$ after the first perturbation. The phase-resetting \\ character of the chemical oscillators allows a detailed analysis, offering insights into the origin of the echo \\ in terms of an intricate structure of phase relationships. Groups of oscillators experiencing different \\ perturbations are analyzed with a geometric approach and in an analytical theory. The characterization of \\ echo phenomena in populations of chemical oscillators reinforces recent theoretical studies of the behavior \\ in populations of phase oscillators [E. Ott et al., Chaos 18, 037115 (2008)]. This indicates the generality of \\ the behavior, including its likely occurrence in biological systems.
}

DOI: 10.1103/PhysRevX.6.041054

Subject Areas: Chemical Physics, Nonlinear Dynamics

\section{INTRODUCTION}

Populations of coupled oscillators display dynamical behavior that is relevant to many natural and manmade systems [1]. Many of the properties of oscillator systems can be captured by using a phase-model approach, originally introduced by Winfree and Kuramoto [2,3]. In a recent study [4], echo behavior was discovered and characterized in populations of phase oscillators. Echo behavior has been studied in a number of fields in physics, in which a nonlinear system subjected to two successive pulses, separated by time $\tau$, shows a measurable response, or "echo," at a time $2 \tau$ after the first perturbation [5]. Example systems include nuclear magnetic resonance, cavity quantum electrodynamics, and plasma waves [6-10].

An interesting aspect of echo phenomena is that macroscopic evidence of the application of the pulses is absent immediately after the decay of the response to the second pulse. Thus, the appearance of the echo at time $2 \tau$ implies that the system retains memory of the past application of the two pulses, even though macroscopically this information appears to have been lost. The system retention of the information resides in the microscopic state through an intricate structure of oscillator phase relationships that is not apparent macroscopically.

Published by the American Physical Society under the terms of the Creative Commons Attribution 3.0 License. Further distribution of this work must maintain attribution to the author(s) and the published article's title, journal citation, and DOI.
The treatment in Ref. [4] is of particular interest because it is potentially applicable in a very general setting relevant to often-encountered situations of many coupled oscillators. This includes applications in biology (e.g., in collections of oscillating yeast cells [11]), ecology (e.g., groups of periodically flashing fireflies $[12,13]$ or croaking frogs [14]), mechanics (e.g., many coupled metronomes [15]), etc. Thus, all these types of systems should be amenable to experimental realization of echo phenomena. Moreover, realization of this goal in one of these settings opens the possibility of employing echoes in system diagnostics, e.g., determination of the underlying distribution function of the oscillator frequencies from a macroscopic measurement (Sec. V).

In this paper, in order to illustrate the wide potential applicability of echo phenomena to large systems of coupled oscillators, we present the first demonstration of echoes in a chemical system: populations of photosensitive Belousov-Zhabotinsky (BZ) chemical oscillators [16-18]. We develop a geometric analysis that allows an understanding of how the echo is manifested through the phaseresetting properties of the system. Furthermore, we show that while the global order parameter is an important tool for demonstrating memory in the system, richer structural (phase-frequency) information is embedded within the separate subpopulations of oscillators associated with different responses to the two perturbations. Finally, we present a general analytical theory for echo phenomena and apply it in a specific analysis of the chemical oscillator system. 


\section{EXPERIMENTS}

Preliminary computational studies revealed that a large number of oscillators would be required to observe the echo phenomenon in the photosensitive BZ system. We therefore developed an oscillator array that permits the simultaneous perturbing and coupling of over 1000 oscillators. (Our previous studies of photochemically coupled BZ oscillators have been limited to approximately 40 oscillators [19-23].) In the oscillator array, the photosensitive oscillators are catalyst-loaded beads of about $200 \mu \mathrm{m}$ in diameter, each of which is fixed on a film of polydimethylsiloxane (PDMS), which is then positioned in an open reactor that is continually replenished with a catalyst-free BZ solution.

Chemistry. - The BZ oscillators are individually monitored with a CCD camera, where the gray level is proportional to the concentration of the oxidized catalyst, $\mathrm{Ru}(\mathrm{bpy}) 3_{3}^{3+}$, and they are illuminated with a spatial light modulator (SLM) for a prescribed duration and intensity. The illumination generates the excited form of the reduced catalyst, $\mathrm{Ru}(\mathrm{bpy})_{3}^{2+*}$, which initiates a sequence of photochemical reactions that produce the autocatalyst $\mathrm{HBrO}_{2}$ $[19,22,24,25]$.

Coupling and perturbations. - The background illumination $\phi_{0}$ is chosen so that the catalyst beads are in an oscillatory state. The photosensitive BZ system allows global coupling of the oscillators by a simple feedback scheme, with the projected light intensity given by $\phi_{j}=\phi_{0}+k\left(\bar{I}-I_{j}\right)$, where $k$ is the coupling strength, $I_{j}$ is the transmitted light intensity of oscillator $j, \bar{I}$ is the average transmitted light intensity of all $N$ oscillators, and $\phi_{j}$ is the projected light intensity on oscillator $j$. Perturbations are applied by increasing the light intensity to $\phi_{p}$ for $3.4 \mathrm{~s}$. During an experiment, the first perturbation is applied $168 \mathrm{~s}$ after introducing the coupling. The second perturbation is then applied $\tau$ seconds later, with $\tau$ chosen to be between 6 and 10 times the mean oscillator period.

Oscillator phase and macroscopic order parameter.We characterize the state of each oscillator $j$ by a phase variable $\theta_{j}=\left(t-t_{n}\right) /\left(t_{n}-t_{n-1}\right)$, where $t_{n}<t$ denotes the time closest to $t$ at which $I_{j}$ is maximum and $n+1$ denotes the number of maxima in the time interval $(0, t)$. (Note that the phase is defined so that $\theta \rightarrow \theta+1$ corresponds to one oscillation.) We obtain the global Kuramoto order parameter [3], $R(t)=$ $N^{-1}\left|\sum_{j} \exp \left(2 \pi i \theta_{j}\right)\right|$, which may be thought of as macroscopically quantifying the degree of synchronization of the oscillator population, in terms of the individual oscillator phases. The order parameter $R(t)$ as a function of time is shown in Fig. 1(a) for a typical experiment with $k=0$. At the time of each perturbation, there is a large increase in the order parameter as the oscillators are phase synchronized. We note that the duration of the applied perturbation in Fig. 1 is small relative to the mean period and the delay time $\tau$.

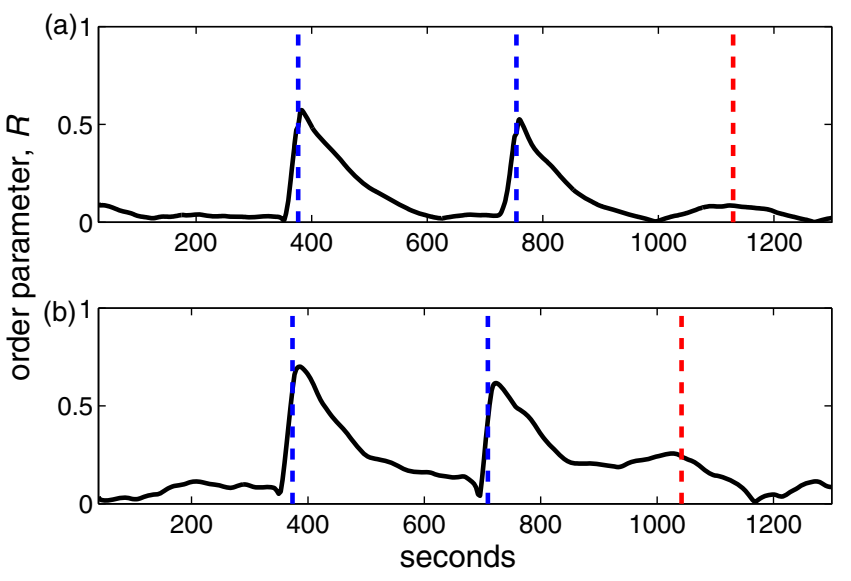

FIG. 1. Experimental measurements illustrating echo behavior in populations of Belousov-Zhabotinsky (BZ) oscillators. The order parameter $R$ is calculated from the phases of the oscillators and plotted as a function of time. The phases of the oscillators are determined using linear interpolation between consecutive peak times. An echo is exhibited in the magnitude of the order parameter $R$ at time $t_{p}+2 \tau$ for a system perturbed at times $t_{p}$ and $t_{p}+\tau$. (a) Uncoupled system, $k=0$, with $t_{p}=376 \mathrm{~s}$, $\tau=378 \mathrm{~s}$, and $N=1295$. (b) Coupled system, $k=0.25$, with $t_{p}=373 \mathrm{~s}, \tau=336 \mathrm{~s}$, and $N=1001$. In these experiments, the critical coupling strength for the onset of synchronization is $k_{c}=0.70$. Average natural period and standard deviation: (a) $T_{0}=36.5 \pm 3.2 \mathrm{~s}$ and (b) $T_{0}=42.6 \pm 8.2 \mathrm{~s}$.

Echo.-Following each perturbation, the frequency heterogeneity gives rise to phase dispersion of the oscillators, leading to a decrease in the order parameter. The echo is observed at time $2 \tau$ after the first perturbation as a spontaneous increase in the order parameter. Echo behavior in an experiment with weak coupling is shown in Fig. 1(b). As is expected, the phase dispersion of the coupled oscillators is now slower following each perturbation. An echo is again observed at time $2 \tau$ following the first perturbation.

\section{SIMULATIONS}

Insights into the echo phenomenon can be gained through simulations of the experimental system. The system is modeled using the Zhabotinsky-BucholtzKiyatkin-Epstein (ZBKE) model [26], modified to describe the photosensitive, discrete BZ oscillator system [19-21]. Each oscillator is described by the concentrations of two key species, $\mathrm{HBrO}_{2}$ and the oxidized form of the catalyst, $\mathrm{Ru}(\mathrm{bpy})_{3}^{3+}$ [27]. An approximately Gaussian frequency distribution in the oscillator population is produced by varying the stoichiometric factor in the ZBKE model for the individual oscillators. In the photoexcitable model, an increase in light intensity leads to increased production of both bromous acid, $\mathrm{HBrO}_{2}$, and the oxidized form of the catalyst, $\mathrm{Ru}(\mathrm{bpy})_{3}^{3+}$. The photochemical coupling is based on the concentration of $\mathrm{Ru}(\mathrm{bpy})_{3}^{3+}$, which corresponds to 
the transmitted light intensity of the micro-oscillators in the experimental system. Simulations are carried out using up to 100,000 oscillators, with the mean period of the oscillators and standard deviation $(42.5 \pm 2.4)$ set to reflect values seen in typical experiments. The simulations also allow exploration of the echo behavior for much larger values of the time delay $\tau$, which is not possible in the experiments because of the gradual depletion of the immobilized catalyst $\mathrm{Ru}(\mathrm{bpy}){ }_{3}^{3+}$.

Figure 2(a) shows the order parameter $R$ as a function of time from simulations of an uncoupled system $(k=0)$. The system was perturbed by momentarily increasing the light intensity at $t_{p}=500$ and again at $\tau=650$ later. Each perturbation is followed by rapid phase dispersion, with a corresponding decrease in the global order parameter. An increase in the order parameter is exhibited at time $t_{p}+2 \tau$, the echo, and a smaller second echo can be seen at time $t_{p}+3 \tau$. The second echo, which is predicted theoretically [4], is typically not visible in the experiments because of experimental noise, although we have observed it on occasion. The value of $\tau$ used in the simulation is approximately 15 times the mean period of the oscillators. Figure 2(b) shows that the echo phenomenon also occurs with a much larger value of $\tau$, equal to approximately 941 periods. We note that this increase in the value of $\tau$ by a factor of about 63 does not significantly affect the magnitude of the echo.

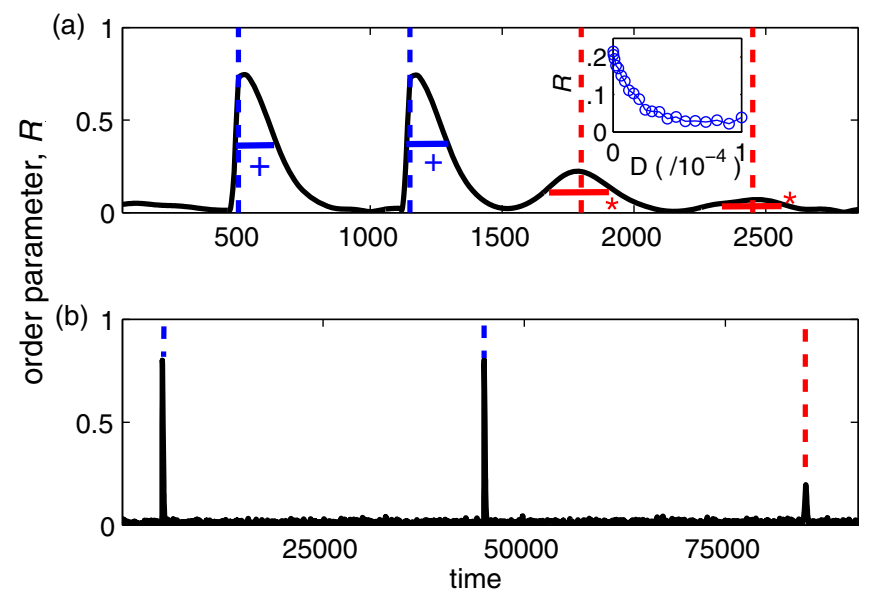

FIG. 2. Simulations of the modified ZBKE model [19-21,26] illustrating echo behavior in populations of uncoupled photosensitive BZ oscillators. The order parameter $R$ is calculated from the phases of the oscillators and plotted as a function of time. An echo is exhibited in the magnitude of $R$ at time $t_{p}+2 \tau$ for a system perturbed at times $t_{p}$ and $t_{p}+\tau$. (a) System with $k=0$, $t_{p}=500, \tau=650, \phi_{p}=0.147$, and $N=5000$. Half width at half maximum for first and second perturbations $(+)$ and width at half maximum for first and second echoes $(*)$. Inset: Maximum value of $R$ at the time of the echo as a function of the noise intensity $D$. (b) System with $k=0, t_{p}=5000, \tau=40000$, $\phi_{p}=0.147$, and $N=5000$. Average natural period and standard deviation $T_{0}=42.5 \pm 2.4$ (dimensionless time units).
Any experimental system has inherent noise, including our system of photosensitive BZ oscillators. The impact of noise on the echo behavior can be explored in the numerical system by introducing additive, independent, Gaussian white noise to each oscillator in the simulation, with noise intensity $D[28,29]$. The inset in Fig. 2(a) shows that the effect of increasing the noise intensity is to decrease the magnitude of the echo. At larger magnitudes of noise, the echo phenomenon is no longer observed. For a fixed, nonzero noise level, the size of the echo decreases with increasing $\tau$, as shown in Ref. [4], since the effect of the noise is accumulated over longer time intervals. It is interesting to note that the decrease of echo strength with increasing separation from the second perturbation has been proposed as the basis of a diagnostic tool for measuring the intrinsic collisional fluctuations (noise) in plasmas [30-32].

Figure 3(a) reveals that, as in the experimental system, the introduction of coupling leads to slower phase dispersion of the oscillators following each perturbation. The impact of the coupling strength on the magnitude of the order parameter at the time of the echo is shown in Fig. 3(b). The magnitude of the echo is an increasing function of $k$. At larger values of $k$, however, the phase dispersion is sufficiently slow that the oscillators cannot fully disperse by the time of the echo, which interferes with the echo phenomenon.

Another factor impacting the magnitude of the echo is the size of the perturbation, as shown in Fig. 4(a). The horizontal axis of this figure is a quantity denoted $X$ (defined subsequently) that increases monotonically with the size of the perturbation. The vertical axis is the
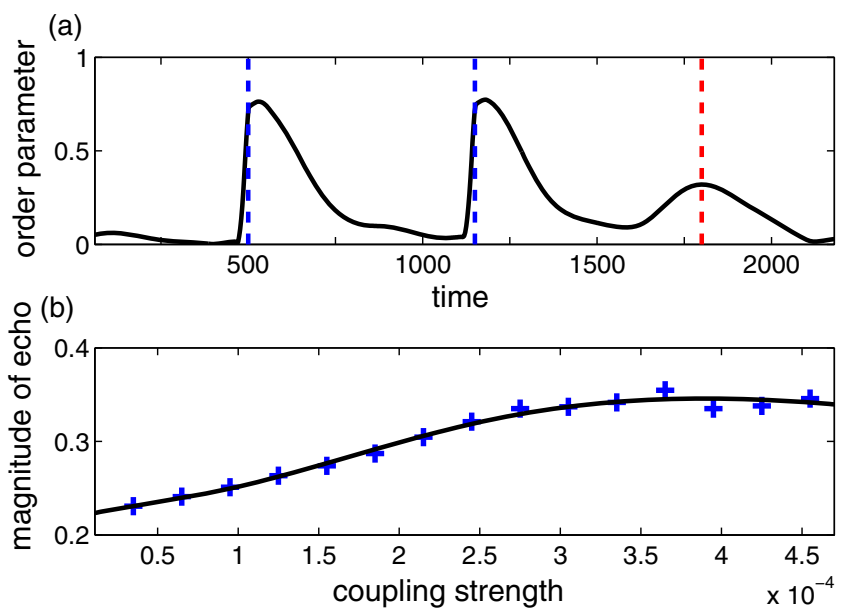

FIG. 3. (a) Numerical simulation of a coupled system with $k=3.35 \times 10^{-4}, \tau=650, \phi_{p}=0.147$, and $N=5000$. Note the slower phase dispersion following each perturbation and the slightly larger magnitude of the echo. (b) Maximum value of the order parameter $R$ at the time of the echo as a function of coupling strength from simulations. The critical coupling strength for the onset of synchronization is $k_{c}=3.0 \times 10^{-3}$ for the simulations in (a) and (b). 
maximum value of the order parameter at the time of the echo from our simulations.

\section{PHASE ANALYSIS}

\section{A. Phase resetting}

In order to explain the definition of the quantity $X$, as well as provide a basis for our later analysis, we now introduce the phase response curve (PRC) [33], which is a plot of the change in phase $\Delta \theta$ on perturbing an isolated oscillator as a function of the preperturbation phase $\theta$. Figure 4(b) shows two PRCs constructed from simulations using large and small perturbations. As shown in the figure, we define $X$ as the size of the phase-resetting region, indicated in both graphs as the maximum possible phase advance. Since the size of the phase-resetting region is a monotonically increasing function of the perturbation size, we are effectively illustrating the size of the echo as a function of the perturbation size in Fig. 4(a). This plot shows a maximum echo response for a value of $X=0.6$.

To gain further insights into the echo phenomenon from our simulations, we plot the phase of each oscillator as a function of its frequency at various times during the simulation. As shown in Fig. 5(a), prior to the first perturbation, the oscillators (plotted as blue dots) are distributed randomly in phase. Immediately following the first perturbation, the oscillators that were in the resetting region of their phase are phase reset to zero, Fig. 5(b), where the reset oscillators are plotted as red dots overlying the unreset oscillators plotted as blue dots. The phases of each of the oscillators that were perturbed, as well as the phases of the oscillators that were not perturbed, then evolve as $\omega_{i}\left(t-t_{p}\right)$, where $\omega_{i}$ is the frequency of the $i$ th oscillator. Oscillators with higher frequencies advance faster, and the phases form a set of points lying along a band, the steepness of which increases with time. At any arbitrary time between the first and second perturbations, the phases of the oscillators lie along a series of offset band segments due to wrapping of the phases (from 1 to 0 ), as shown in Fig. 5(c). The steepness and number of the bands increase with time, while their thickness decreases. This can be seen in Fig. 5(c), which corresponds to the time halfway between the two perturbations, compared to Fig. 5(d), which corresponds to the time immediately prior to the second perturbation. At the time of the second perturbation, $t_{p}+\tau$, any oscillator lying in the resetting region is phase reset to zero, as shown in Fig. 5(e), where the red (blue) oscillators of Fig. 5(d) that are reset are plotted in Fig. 5(e) with their color changed from red (blue) to green (black). At the time of the echo, $t_{p}+2 \tau$, the oscillators are distributed along either a set of thin bands or a set of short steeper band segments that appear to branch off the primary bands, Fig. 5(f). Note that the four colors appearing in Figs. 5(e) and 5(f) correspond to four distinct groups of oscillators: Group I (green) contains those oscillators that are reset by both perturbations; group II (red) contains those oscillators that are reset by the first but not the second perturbation; group III (black) contains those oscillators that are reset by the second but not the first perturbation; group IV (blue) contains those oscillators that are not phase reset by either perturbation.

Figure 6 shows the equivalent phase vs frequency plots for the individual oscillators in the experimental system. While there is evidently noise present in the system, the same underlying features as in the numerical simulation are clearly visible. A certain fraction of the initially randomly distributed oscillators, Fig. 6(a), are phase reset by the first perturbation, Fig. 6(b). The oscillators then
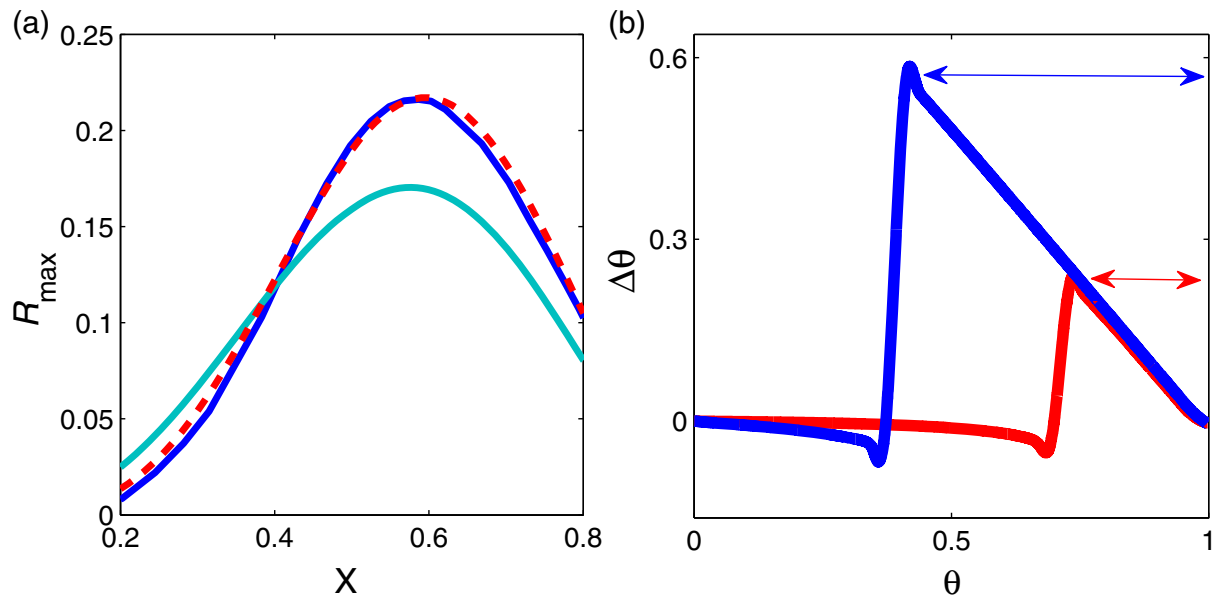

FIG. 4. (a) Magnitude of the order parameter $R$ at the time of the echo as a function of the size of the phase-resetting region $X$, which increases monotonically with the size of the perturbation. We show ZBKE simulation values (dark blue line) and values calculated with the geometric approach using groups I and II (light blue line). The red dashed line shows values calculated with the geometric approach using groups I-IV or with Eq. (16) from the analytical theory. (b) Phase response curves for stimuli $\phi_{p}=0.147$ (blue line) and $\phi_{p}=0.0218$ (red line) from ZBKE simulations. The sizes of the associated phase-resetting regions $X$ are shown by the red and blue horizontal arrows, respectively. Simulation parameters are as in Fig. 2. 

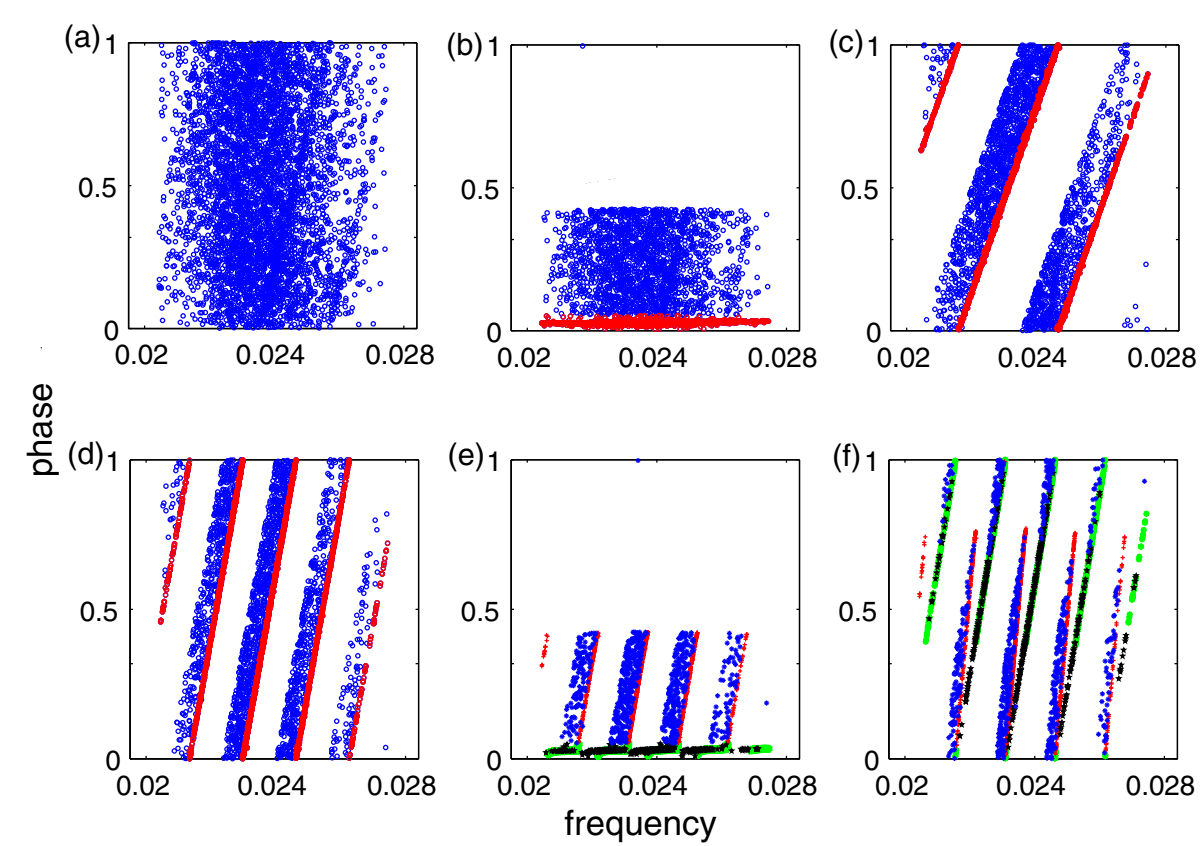

FIG. 5. Plots of phase as a function of frequency of oscillators in the ZBKE simulation shown in Fig. 2(a) at the following times: (a) immediately prior to the first perturbation at $t_{p}$, (b) immediately following the first perturbation, in which oscillators in the resetting region of their phase are phase reset to 0, (c) halfway between the two perturbations, $t_{p}+\tau / 2$, (d) immediately prior to the second perturbation, (e) immediately following the second perturbation, in which oscillators in the resetting region of their phase are phase reset to 0 , and (f) at the time of the echo, when a series of narrow bands is observed, each with a single branch segment. The phase-resetting region at each perturbation is given by the phase range $(1-X)$ to 1 , where $X$ is the size of the region as defined in Fig. 4(b). Here, $X=0.6$ and the phase-resetting region spans the phase interval $[0.4,1)$. Parameters: $\tau=650$. Other parameters as in Fig. 2. See text for color coding of the oscillators.

evolve into a series of bands that steepen with time, Figs. 6(c) and 6(d). A certain fraction of the oscillators are phase reset at the second perturbation, Fig. 6(e), and banding structure is again visible at the time of the echo, Fig. 6(f).

\section{B. Structure of phase distribution}

Examination of Figs. 5(f) and 6(f) reveals that there is structure and information within the phase distribution of the oscillators at the time of the echo. How this arises, however, and why it leads to an increase in the order
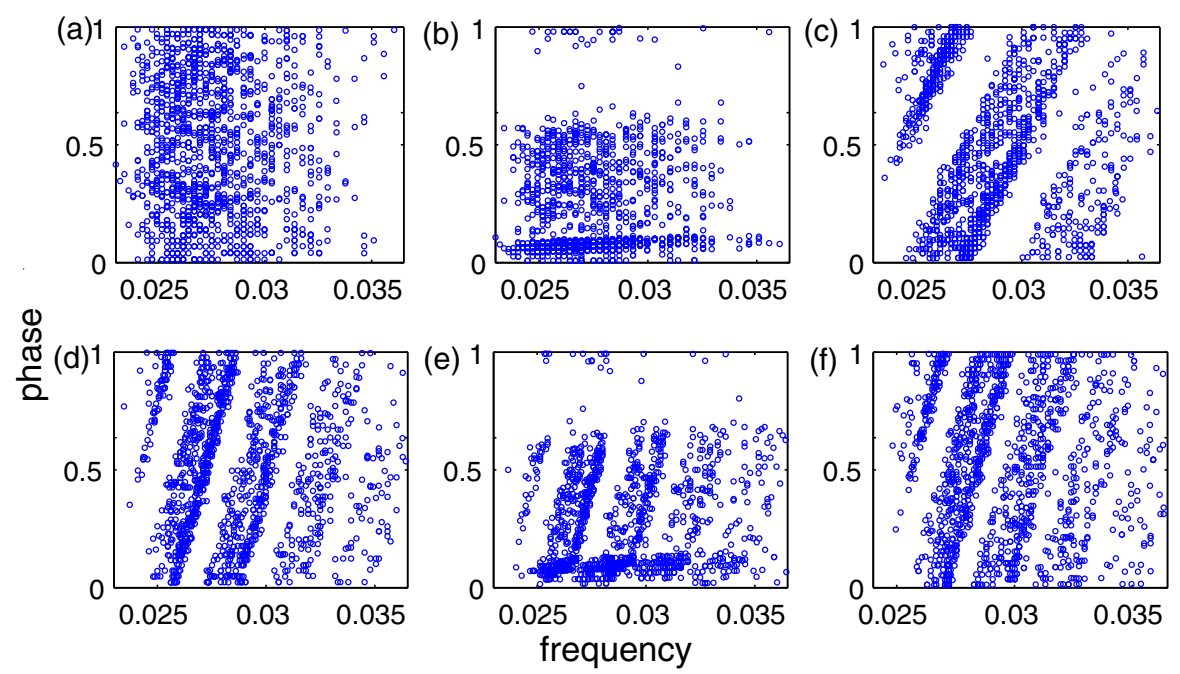

FIG. 6. Plots of phase as a function of frequency of oscillators from the experiment shown in Fig. 1(a) at the following times: (a) immediately prior to the first perturbation at $t_{p}$, (b) immediately following the first perturbation, in which oscillators in the resetting region of their phase are phase reset to 0 , (c) halfway between the two perturbations, $t_{p}+\tau / 2$, (d) immediately prior to the second perturbation, (e) immediately following the second perturbation, in which oscillators in the phase range $(1-X)$ to 1 are phase reset to 0 , and (f) at the time of the echo. Note that there is no color coding, as in Fig. 5, of the oscillator groups I-IV. 
parameter at time $t_{p}+2 \tau$ is not immediately apparent. To gain a better understanding, we focus on the numerical simulations and the division of the oscillator populations into four groups as previously discussed. By tracking the behaviors of groups I and II, we find that much of the underlying structure of the echo can be understood since groups III and IV make a smaller contribution to the size of the order parameter at the time of the echo. Thus, we first focus on groups I and II. Assuming that the oscillators are uniformly distributed in phase at the time of the perturbation, we can approximate $X$ in Fig. 4(a) as the fraction of all of the oscillators that are phase reset by a perturbation. The occupancies of groups I and II are then estimated to be $N X^{2}$ and $N X(1-X)$, respectively.

Figure 7 shows plots of phase as a function of frequency of the oscillators belonging to groups I and II at different times during the simulation. Just prior to the first perturbation, the oscillators of both of these groups are, by definition, found between phases $(1-X)$ and 1 , as shown in Figs. 7(a)(i) and 7(b)(i). Because of the random phases at $t=0$ used in the simulation, there is no dependence of phase on frequency. Both sets of oscillators are phase reset to zero by the first perturbation. At the time of the second perturbation, the line segments in group II mutually align in the refractory region of the phase, as shown in Fig. 7(b)(ii). In contrast, the line segments associated with the group I oscillators align in the resetting region of the phase and are phase reset to 0 by the second perturbation, as shown in Fig. 7(a)(ii).

At the time of the echo, the group I oscillators have exactly the same phases as immediately prior to the second perturbation, with the oscillators arranged along a series of mutually aligned line segments in the resetting region of the phase, as shown in Fig. 7(a)(iii). The group II oscillators, which were not phase reset at the second perturbation, are now aligned along a series of line segments that are steeper than and twice as long as they were at the time of the second perturbation, Fig. 7(b)(iii).

In Fig. 7, there are gaps in the observed frequencies of both groups, with the missing frequencies in the group I plots corresponding to the frequencies present in the group II plots, and vice versa. This occurs because the definition of each group places separate restrictions on the allowed frequencies of its member oscillators. For example, oscillators in group II are defined as those that are phase reset by the first perturbation but not the second; i.e., their phases must lie between 0 and $(1-X)$ at time $t_{p}+\tau$. Since they start at zero phase at time $t_{p}$, the allowed frequencies of the oscillators are given by $n / \tau+\xi / \tau$, with $\xi$ restricted to the range $0 \leq \xi<(1-X)$, where $n$ can take any integer value. The separate line segments in Fig. 7(b)(ii) correspond to increasing values of $n$, with the oscillators arranged along the central line segment having the range of frequencies corresponding to $n=15$. This is in agreement with the value of $\tau$ for this simulation, being approximately 15 times the value of the mean period of the oscillators. The group I oscillators have frequencies $n / \tau+\xi / \tau$, with $\xi$ restricted to the range $(1-X) \leq \xi<1$. This ensures that they lie within the resetting region at the time of the second perturbation.

With this formulation of the allowed frequencies, we can understand why the oscillators in group II are distributed along longer and steeper line segments at time $2 \tau$. An arbitrary oscillator in group II at time $\tau$ following the first perturbation will have phase $\xi$, while at time $2 \tau$ following the first perturbation, its phase will be $2 \xi$. For both groups, these allowed frequencies also ensure that the line segments associated with each value of $n$ will mutually align at integer multiples of time $\tau$ following the first perturbation.

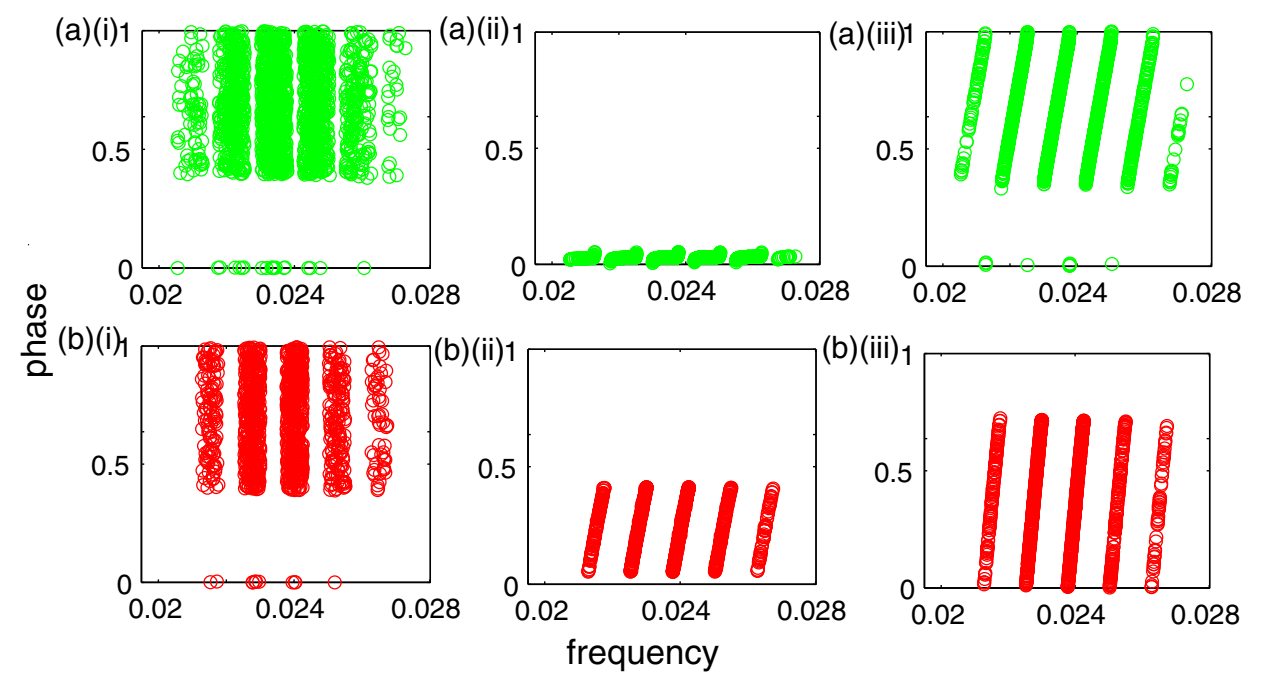

FIG. 7. Phase as a function of frequency of oscillators from a ZBKE simulation for (a) group I oscillators and (b) group II oscillators at the following times: (i) immediately prior to the first perturbation, (ii) immediately following the second perturbation, and (iii) at the time of the echo. All parameters are as in Fig. 2. 


\section{Geometric phase analysis}

The value of the order parameter at the time of the echo can now be estimated based on the phase distributions of the group I-IV oscillators. The shapes of the distributions are determined by geometric restrictions on the phasefrequency relationships of each group. For example, the phase distribution of the oscillators in group I is simply the rectangle corresponding to the phase-resetting region, as defined in Fig. 4(b) and illustrated in Fig. 5(b). As shown in Fig. 8(a), the group I oscillators are found between $(1-X)$ and 1, while the group II oscillators are found between 0 and $2(1-X)$. Our approach assumes that the frequencies of the oscillators in a given group are uniformly distributed through their respective regions immediately prior to each perturbation, which is valid for large $N$. The actual distributions determined from ZBKE simulations, shown in Fig. 8(a), verify the validity of this assumption.

The order parameter calculated using the phase distributions of groups I and II as a function of $X$ is shown in Fig. 4(a) by the light blue curve. The estimate of the order parameter can be improved by including the impact of groups III and IV. The phase distributions of these oscillators at the time of the echo are also determined using a geometric approach, which is described in Appendix A. Figure 8(a) shows these geometrically determined distributions along with the distributions from ZBKE model simulations.

The overall phase distribution of all oscillators is shown in Fig. 8(a), where the dominant asymmetry, which leads to the larger value of $R$, arises from the overlap of oscillators from groups I and II, with smaller contributions from groups III and IV. Figure 4(a) shows remarkably good agreement between the magnitude of the geometrically determined order parameter at the time of the echo as a function of $X$, shown by the red dashed curve, with that calculated using the ZBKE model.

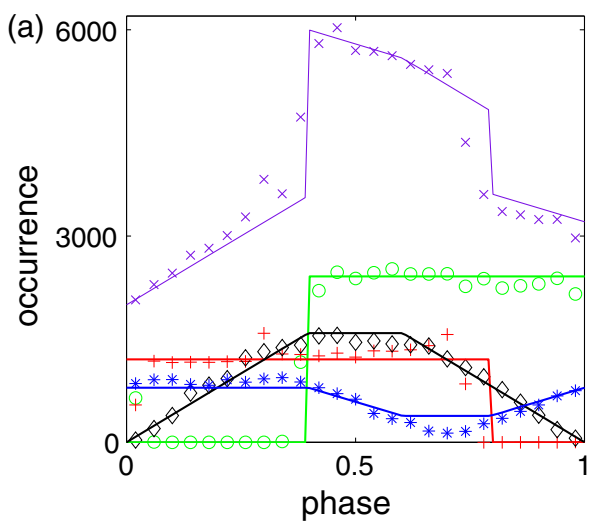

A close-up of the central branched band described earlier in Fig. 5(f) is shown in Fig. 8(b), color coded by the four groups. The members of groups I and II are seen to span the entire range of allowed frequencies, i.e., between $14 / \tau$ and $15 / \tau$, as discussed above. Member oscillators of these two groups are uniformly distributed along their respective line segments. Group III oscillators are also distributed along a thin line, although the distribution is now not uniform. Figure 8(a) shows the geometrically estimated (black line) and simulation-determined (black symbols) density distributions. The unperturbed oscillators of group IV are restricted to certain regions of this phase-frequency plot. These ranges arise, as for groups I-III, from frequency and initial phase restrictions on the oscillators that permit them to be a member of the group.

\section{THEORETICAL ANALYSIS OF ECHO BEHAVIOR IN THE CASE OF NOISE-FREE UNCOUPLED OSCILLATORS}

To make the theoretical treatment tractable, we consider the case where there is no coupling between the oscillators, but, unlike the theoretical treatment in Ref. [4], we do not take the externally applied perturbations to be small. For convenience, we assume that two equal-amplitude external pulse perturbations (unequal amplitudes can be easily incorporated), each of negligible duration, are applied at time $t=0$ and at time $t=\tau$, and we wish to evaluate the order parameter response, $R(t)=N^{-1}\left|\sum_{j} \exp \left(2 \pi i \theta_{j}\right)\right|$, in the limit where the number of oscillators $N$ is large. As in Sec. IV, we assume that the action of a pulse perturbation can be described by a phase response curve, such that if the phase of an oscillator is $\theta_{-}$just before application of a pulse perturbation, then its phase $\theta_{+}$just after application of the pulse perturbation is

$$
\theta_{+}=\rho\left(\theta_{-}\right)
$$

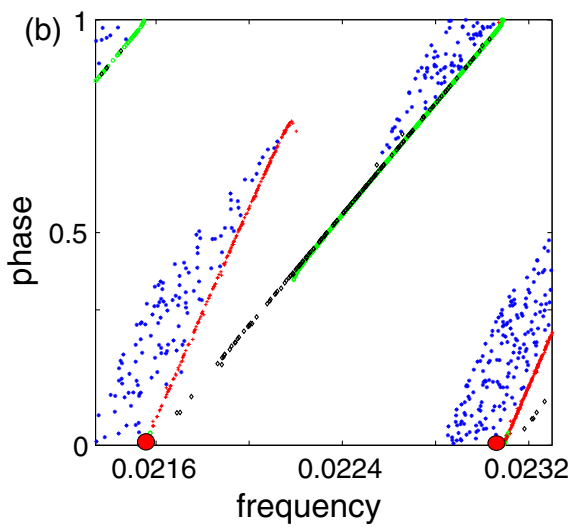

FIG. 8. (a) Geometrical prediction of phase distribution of oscillators (solid lines) and ZBKE simulation values (symbols) at the time of the echo. Green (open circle), red (plus), black (diamond), and blue (star) symbols and lines correspond to groups I, II, III, and IV, respectively. The violet symbols (times) and line correspond to the sum of the distributions of the four groups. The ZBKE simulations were carried out with a large number of oscillators, $N=100000$. (b) Detail of structure from Fig. 5(f), color coded according to the four different groups in (a) at the time of the echo. The frequencies indicated by the left and right filled red circles correspond to $14 / \tau$ and $15 / \tau$, respectively. Only half of the population of group III oscillators (black) are plotted in order to not obscure the group I oscillators (green). 
where a plot of $\Delta\left(\theta_{-}\right)=\left(\theta_{+}-\theta_{-}\right) \bmod 1$ versus $\theta_{-}$is the phase response curve (PRC) [recall that we use the notation $0 \leq \theta<1$; i.e., the interval $[0,1)$ corresponds to one complete cycle of an oscillation]. In the absence of a pulse, the phase $\theta_{j}(t)$ of oscillator $j$ is governed by $\theta_{j}=\hat{\theta}_{j}$ modulo $1, d \hat{\theta}_{j}(t) / d t=f_{j}$. Thus,

$$
\theta_{j}(t)= \begin{cases}\left(\rho\left(\theta_{0 j}\right)+f_{j} t\right) \text { modulo } 1 & \text { for } 0<t<\tau \\ \left\{\rho\left[\rho\left(\theta_{0 j}\right)+f_{j} \tau\right]+f_{j}(t-\tau)\right\} \text { modulo } 1 & \text { for } t>\tau,\end{cases}
$$

where $\theta_{0 j}=\theta_{j}\left(0^{-}\right)$is the oscillator phase just before application of the first perturbing pulse. The initial phases $\theta_{0 j}(j=1,2, \ldots, N)$ are assumed to be uniformly distributed in $[0,1)$. Appropriate to the large- $N$ limit, we drop the subscripts $j$ on $\theta_{j}$ and $f_{j}$, and express the complex order parameter $\hat{R}(t)=N^{-1} \sum_{j} \exp \left(2 \pi i \theta_{j}\right)$ response for $0<t<\tau$ as

$\hat{R}(t)=\int_{0}^{1} d \theta_{0} \int_{-\infty}^{+\infty} d f G(f) \lambda\left(\theta_{0}\right) e^{2 \pi i f t}, \quad$ for $0<t<\tau$,

where $G(f)$ is the oscillator distribution function $\left(\int_{-\infty}^{+\infty} G(f) d f=1\right)$, and

$$
\lambda\left(\theta_{0}\right)=\exp \left[2 \pi i \rho\left(\theta_{0}\right)\right] .
$$

For $t>\tau, \theta(t)=\theta\left(\tau^{+}\right)+f(t-\tau)$, where $\tau^{+}$denotes the time instant just after the second pulse perturbation. Thus, we can express $\hat{R}(t)$ for $t>\tau$,

$\hat{R}(t)=\int_{0}^{1} d \theta_{0} \int_{-\infty}^{+\infty} d f G(f) \eta\left(\theta_{0}, f \tau\right) e^{2 \pi i f t}, \quad$ for $t>\tau$,

where $\eta=\exp \left\{2 \pi i\left[\theta\left(\tau^{+}\right)-f \tau\right]\right\}$, or

$$
\eta\left(\theta_{0}, f \tau\right)=\exp \left\{2 \pi i\left[\rho\left(\rho\left(\theta_{0}\right)+f \tau\right)-f \tau\right]\right\} .
$$

From Eqs. (3) and (4), we obtain

$$
\hat{R}(t)=A \tilde{G}(t), \quad \text { for } 0<t<\tau,
$$

where

$$
A=\int_{0}^{1} \lambda\left(\theta_{0}\right) d \theta_{0}
$$

and $\tilde{G}$ denotes the inverse Fourier transform of the frequency distribution function $G(f)$,

$$
\tilde{G}(t)=\int_{-\infty}^{+\infty} G(f) e^{2 \pi i f t} d f .
$$

For example, if $G(f)$ is a Gaussian distribution with mean $\bar{f}$ and variance $(\Delta f)^{2}$,

$$
G(f)=\frac{1}{\sqrt{2 \pi} \Delta f} \exp \left\{-(f-\bar{f})^{2} /\left[2(\Delta f)^{2}\right]\right\}
$$

then

$\tilde{G}(t)=\exp \left\{-\frac{1}{2}(2 \pi \Delta f t)^{2}+2 \pi i \bar{f} t\right\}, \quad$ for $t>0$.

Thus, the initial response to the first pulse perturbation is confined to a time interval of order $(\Delta f)^{-1}$.

Letting $\xi=f \tau$ and noting from Eq. (6) that $\int_{0}^{1} \eta\left(\theta_{0}, \xi\right) d \theta_{0}$ is periodic with period one in $\xi$, we expand it in a Fourier series,

$$
\int_{0}^{1} \eta\left(\theta_{0}, \xi\right) d \theta_{0}=\sum_{q=-\infty}^{+\infty} C_{q} \exp (-2 \pi i q \xi),
$$

where

$$
C_{q}=\int_{0}^{1} d \theta_{0} \int_{0}^{1} d \xi e^{2 \pi q i \xi} \eta\left(\theta_{0}, \xi\right)
$$

Inserting Eq. (11) into Eq. (5), we obtain $\hat{R}(t)$ for $t>\tau$,

$$
\hat{R}(t)=\sum_{q=-\infty}^{+\infty} C_{q} \tilde{G}(t-q \tau) .
$$

Thus, the response to the initial perturbation $[A \tilde{G}(t)$ for $t>0$, cf. Eq. (7)], the immediate response to the second perturbation, the term $C_{1} \tilde{G}(t-\tau)$, for $t>\tau$ [Eq. (13)], and the echoes at times $t=q \tau$ [given by $C_{q} \tilde{G}(t-q \tau), q=$ $2,3, \ldots]$ all have the same shape [Eq. (10)]. For the example of Gaussian $G(f)$, the pulse width at half maximum is

$$
\Delta t=\sqrt{8 \ln 2} /(2 \pi \Delta f) \cong T^{2} \sqrt{8 \ln 2} /(2 \pi \Delta T),
$$

where $\Delta T$ is the spread in the oscillator period $T(\gg \Delta T)$. Assuming that $\Delta t \ll \tau$ (the case of interest), the response to the two pulse perturbations and the individual echo responses are all well separated in time, as illustrated in Fig. 2(a). We find that the standard deviation $\Delta T$ predicted from the half width at half maximum, $\Delta t / 2$, following the first or second perturbation or the full width at half maximum $\Delta t$ for the first or second echo agrees well with the simulation values. For the experiment shown in Fig. 1(a), the predicted standard 
deviation is $3.3 \mathrm{~s}$ compared to the actual value of $3.2 \mathrm{~s}$, and for the simulation shown in Fig. 2(a), the predicted value is 2.5 compared to the actual value of 2.4 . We also note that if the mean period is not known, it can be readily found from the oscillations of the real part of the order parameter, $\operatorname{Re}[\hat{R}(t)]$, following the perturbations or at the echoes.

We emphasis that, in the preceding, we have neglected collective interactions that couple the oscillator phases. Such interactions have been treated in Ref. [4] by the use of perturbation theory (appropriate when the pulse perturbations are small). In the case where oscillator coupling is included [4], the decay of the response following a pulse perturbation or of an echo is typically exponential in time. In contrast, without coupling, depending on $G(f)$, the decay can be faster than exponential, as illustrated by Eq. (10). This is consistent with the slower post-perturbation fall-off seen in Fig. 1(b) (nonzero coupling) as compared to Fig. 1(a) (no coupling) in the experiments and in Fig. 3(a) (nonzero coupling) as compared to Fig. 2(a) (no coupling) in the simulations.

For the case of the BZ reaction PRC [Fig. 4(b)], a good approximation to $\rho(\theta)$ is

$$
\rho(\theta)= \begin{cases}\theta & \text { for } 0 \leq \theta<(1-X) \\ 0 & \text { for }(1-X) \leq \theta<1\end{cases}
$$

In this case, the integral in Eq. (12) can be explicitly evaluated analytically (see Appendix B), with the result

$$
C_{q}= \begin{cases}X\left(b_{q-1}-b_{q}\right)-\left|b_{q-1}\right|^{2}+b_{q} b_{1-q} & \text { for } q \neq 0,1 \\ (1-X)\left(X-b_{1}\right) & \text { for } q=0 \\ X^{2}-(1-X) b_{1} & \text { for } q=1\end{cases}
$$

where $b_{q}=[1-\exp (-2 \pi i q X)] /(2 \pi i q)$. Using this result for $q=2$ (the first echo response), we obtain the dashed red curve in Fig. 4(a), which shows good agreement with the dark blue curve in Fig. 4(a). The dashed red curve is identical to the curve obtained using the geometric phase analysis described in Sec. IV C. Note that the dark blue curve is obtained using PRCs from ZBKE simulations, as in Fig. 4(b), as opposed to the approximation, Eq. (15), used in both the geometric phase analysis in Sec. IV C and in the results presented here.

\section{CONCLUSION}

Echo behavior is a somewhat counterintuitive phenomenon. The apparent loss of information manifested by the measured decay of the order parameter following a pulse is not due to dissipative, entropy-increasing processes, but instead arises through the inherent phase dispersion. Thus, the information that perturbations have previously occurred remains encoded within the phase structure of the oscillators. The echo phenomenon observed with the macroscopic order parameter, while remarkable, does not in itself reveal the extent of this underlying information. Only a component of the information is interrogated by calculation of the order parameter, with the increase in $R$ at the time of the echo arising from the alignment of the phase-frequency distributions primarily associated with groups I and II.

Noise (which can be regarded as an entropy-increasing process) can lead to the destruction of the relative phase structure of the oscillators. Our simulations show that for a given $\tau$, the size of the echo decreases as the magnitude of noise increases in the system. At sufficiently large magnitudes of noise, the echo no longer occurs. Equivalently, Ref. [4] showed that for a fixed magnitude of noise, the size of the echo decreases with increasing delay time $\tau$. The observation of an echo in our experimental system, Fig. 1, indicates that the system is within the range in which the time-integrated amount of noise is sufficiently small that the encoded phase structure is not destroyed, as demonstrated by Fig. 6. Within this range, as with our numerical simulations subject to small levels of noise, the echo phenomenon is robust.

We have presented the first example of echo behavior in populations of chemical oscillators. Simulations using a realistic model of the photosensitive $\mathrm{BZ}$ micro-oscillators agree well with the experimental behavior. A geometric phase analysis is presented that offers insights into the origin of the echo and provides evidence for an intricate structure of phase relationships underlying the behavior. A general analytical theory is presented for echo phenomena for the case of large perturbations and is applied to accurately predict features of the echo behavior found experimentally and in simulations of BZ oscillator populations. Our study demonstrates that echo behavior is sufficiently robust to occur in real-world settings including biological systems.

\section{ACKNOWLEDGMENTS}

This article is based on work supported by the National Science Foundation (Grant No. CHE-1565665). The work of E. O. was supported by the U. S. Army Research Office (Grant No. WF911NF10101). Desmond Yengi, Razan Snari, and Sadegh Faramarzi Ganjabad of West Virginia University carried out preliminary experiments.

\section{APPENDIX A: GEOMETRIC ANALYSIS OF PHASE STRUCTURE}

We can use a geometric approach to determine the phase distributions of the oscillators associated with group III and IV at the time of the echo. We illustrate the approach by considering the oscillators in group III. At time $t_{p}$, these oscillators are uniformly distributed in the region 0 to $(1-X)$, as illustrated in Fig. 9(a). At time $t_{p}+\tau$, they are uniformly distributed in the region $(1-X)$ to 1 . This places 
restrictions on their allowed frequencies, although, in contrast to groups I and II, the restrictions are now dependent on the particular phase of an oscillator at time $t_{p}$. Consider the oscillators with the narrow range of phases indicated in red in Fig. 9(a). At time $t_{p}+\tau$, these oscillators will lie uniformly in the region $(1-X)$ to 1 . Therefore, the allowed frequencies of oscillators in this thin region are $n / \tau+\xi / \tau$, with $\xi$ restricted to the range $1-X$ to 1 . In Fig. 9(b), we schematically draw the phase location of these oscillators, uniformly spread through the phase-resetting region. Oscillators in the narrow range of phase indicated in light blue in Fig. 9(a) show a phase advance between 0 and $X$. In Fig. 9(b), these oscillators are schematically illustrated as spread through the resetting region at the time of the second perturbation. Their allowed frequencies are given by $n / \tau+\xi / \tau$, with $\xi$ restricted to the range 0 to $X$. For the general narrow region of oscillators at phase $\theta_{0}$ at time $t_{p}$, indicated in green in Figs. 9(a) and 9(b), the value of $\xi$ is restricted to the range $1-X-\theta_{0} \leq \xi<1-\theta_{0}$. At time $t_{p}+\tau$, all of the oscillators are phase reset to 0 , as schematically illustrated in Fig. 9(c). The location of any oscillator $\tau$ later can be determined using the above-allowed frequencies. The oscillators that were in the red region will advance through $(1-X)$ to 1 , whereas the oscillators that were in the light blue region will advance from 0 to $X$. The location of each of these sets of oscillators in phase at the time of the echo is shown in Fig. 9(d), with the red and blue oscillators offset from each other in phase. These sets of oscillators can be thought of as forming the top and bottom

(a)

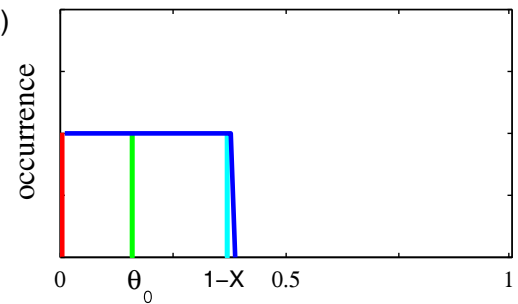

(c)

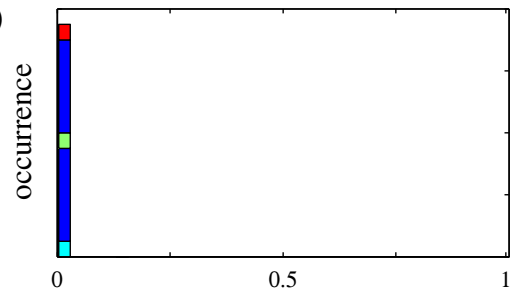

sides of a parallelogram. The location of an arbitrary set of oscillators, which had phases close to $\theta_{0}$ at time $t_{p}$, is bounded by the parallelogram's other sides. The total number of oscillators at a particular phase in Fig. 9(d) is proportional to the internal height of the parallelogram at that phase. By internal height, we mean either the vertical distance from the bottom or top side to its adjacent side, or the parallelogram's altitude. Assuming the altitude of the parallelogram is 1 , in the case where $X \geq 0.5$, the internal height as a function of phase, $\psi$, is equal to $\psi /(1-X)$ for $\psi \leq(1-X), 1$ for $(1-X)<\psi \leq X$ and $(1-\psi) /(1-X)$ for $\psi>X$. In the case where $X<0.5$, the internal height is equal to $\psi /(1-X)$ for $\psi \leq X, X /(1-X)$ for $X<\psi \leq(1-X)$, and $(1-\psi) /(1-X)$ for $\psi>(1-X)$. This is plotted in Fig. 8 (a) as the black line. The phase distribution of the oscillators in group IV at the time of the echo, as shown in Fig. 8(a) as a blue line, can be determined with a similar geometric approach.

\section{APPENDIX B: EVALUATION OF THE ECHO STRENGTH $C_{q}$ GIVEN IN EQ. (16) FOR THE APPROXIMATION TO THE BZ REACTION PRC GIVEN IN EQ. (15)}

From the previously given definitions of the oscillator groups, the oscillator phase angles $\theta(t)$ for groups I, II, III, and IV are given in Table I, where $\theta_{0}$ denotes the uniformly distributed oscillator phases just before application of the first perturbation pulse at $t=0$.

(b)

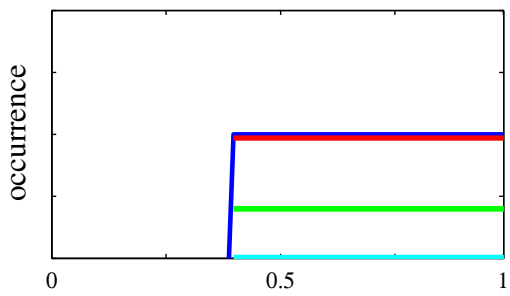

(d)

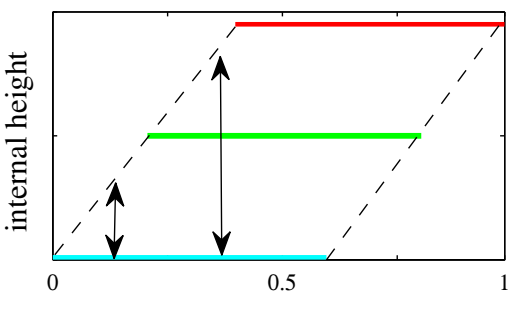

FIG. 9. Schematic representation for the geometric analysis of the phase distribution of group III oscillators. (a) At time $t_{p}$, the oscillators are uniformly distributed through the refractory region. (b) Just prior to the second perturbation, the same oscillators are now uniformly distributed through the resetting region. (c) The oscillators are then phase reset by the perturbation at time $t_{p}+\tau$. The red, light blue, and green regions indicate the oscillators in one of three narrow phase ranges at time $t_{p}$. The order of their vertical stacking in (b) and (c) is arbitrary. Each horizontal band contributes equally to the total number of oscillators at a particular phase. (d) The phase range of each set (red, blue, and green) of oscillators at the time of the echo. Since the choice of phase $\theta_{0}$ is arbitrary, the phase spread of the other oscillators is bound by the sides of the parallelogram (dotted black line). 
TABLE I. Oscillator phase angles $\theta(t)$ for groups I, II, III, and IV.

\begin{tabular}{lll}
\hline \hline Group & \multicolumn{1}{c}{$\theta(t)$ for $0<t<\tau$} & \multicolumn{1}{c}{$\theta(t)$ for $t>\tau$} \\
\hline I & $\theta(t)=(f t) \bmod 1$ & $\theta(t)=[f(t-\tau)] \bmod 1$ \\
II & $\theta(t)=(f t) \bmod 1$ & $\theta(t)=(f t) \bmod 1$ \\
III & $\theta(t)=\left(f t+\theta_{0}\right) \bmod 1$ & $\theta(t)=[f(t-\tau)] \bmod 1$ \\
IV & $\theta(t)=\left(f t+\theta_{0}\right) \bmod 1$ & $\theta(t)=\left(f t+\theta_{0}\right) \bmod 1$ \\
\hline \hline
\end{tabular}

Using Table I and the previously specified definitions of the four groups, we can divide the unit square, $0 \leq \theta_{0}<1$, $0 \leq f \tau$ modulo $1=\xi \leq 1$, into regions associated with each of the four groups, as shown in Fig. 10. Specifically, we arrive at this diagram by noting that the conditions corresponding to the four groups are as follows:

$$
\begin{aligned}
A_{\mathrm{I}}:(1-X) \leq \theta_{0}<1, & (1-X) \leq(f \tau) \bmod 1<1 \\
A_{\mathrm{II}}:(1-X) \leq \theta_{0}<1, & 0 \leq(f \tau) \bmod 1<(1-X) \\
A_{\mathrm{III}}: 0 \leq \theta_{0}<(1-X), & (1-X) \leq\left(f \tau+\theta_{0}\right) \bmod 1<1 \\
A_{\mathrm{IV}}: 0 \leq \theta_{0}<(1-X), & 0 \leq\left(f \tau+\theta_{0}\right) \bmod 1<(1-X) .
\end{aligned}
$$

In the above, the first condition in the pair of conditions for each $A_{K}$ arises from the specification that the first pulse resets (does not reset) the oscillators in $A_{\mathrm{I}}$ and $A_{\mathrm{II}}\left(A_{\mathrm{III}}\right.$ and $A_{\mathrm{IV}}$ ), while the second condition arises by evaluating the expressions for $\theta(t)$ in the $0<t<\tau$ column of Table I at the time $t=\tau$ and then noting that oscillators in $A_{\mathrm{I}}$ and $A_{\mathrm{III}}$ ( $A_{\mathrm{II}}$ and $A_{\mathrm{IV}}$ ) are reset (are not reset) by the second pulse. Region $A_{\mathrm{IV}}$ appears as the union of two subregions, $A_{\mathrm{IV}}^{\prime}$ and $A_{\mathrm{IV}}^{\prime \prime}$, within the unit square of $\xi-\theta_{0}$ space, with $A_{\mathrm{IV}}^{\prime}$ corresponding to $\left(\xi+\theta_{0}\right) \bmod 1=\left(\xi+\theta_{0}\right)$ and $A_{\mathrm{IV}}^{\prime \prime}$ corresponding to $\left(\xi+\theta_{0}\right) \bmod 1=\left(\xi+\theta_{0}-1\right)$. By construction,

$$
\eta\left(\theta_{0}, f \tau\right)=\exp \left\{2 \pi i\left[\theta\left(\tau^{+}\right)-f \tau\right]\right\}
$$

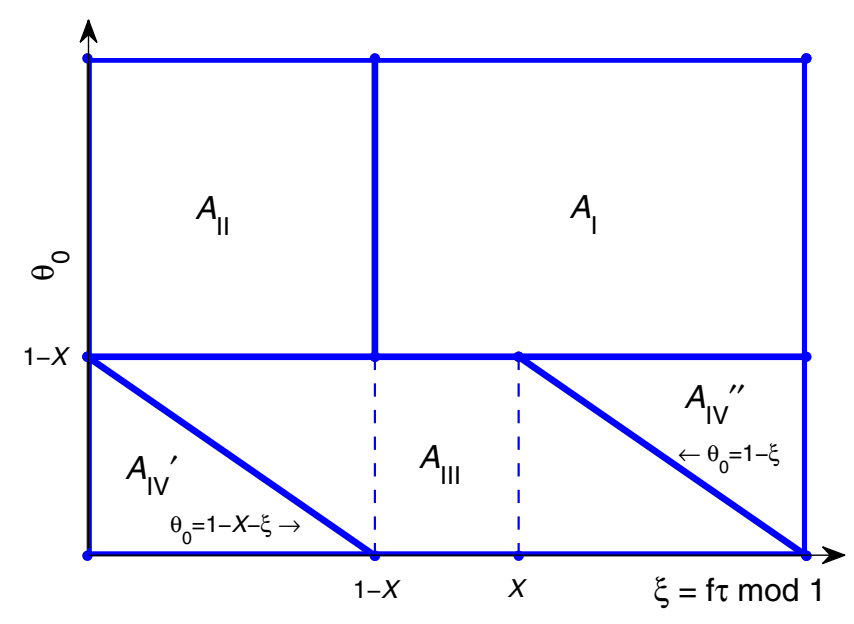

FIG. 10. Values $\left(\xi, \theta_{0}\right)$ in region $A_{K}(K=\mathrm{I}$, II, III, IV) corresponding to oscillators in group $K$. where $\theta\left(\tau^{+}\right)$denotes the oscillator phase at the time instant just after the second pulse perturbation. Thus, from the rightmost column of Table I, evaluated at $t=\tau$,

$\eta\left(\theta_{0}, \xi\right)= \begin{cases}e^{-2 \pi i \xi} & \text { for }\left(\theta_{0}, \xi\right) \text { in region } A_{\mathrm{I}} \\ 1 & \text { for }\left(\theta_{0}, \xi\right) \text { in region } A_{\mathrm{II}} \\ e^{-2 \pi i \xi} & \text { for }\left(\theta_{0}, \xi\right) \text { in region } A_{\mathrm{III}} \\ e^{2 \pi i \theta_{0}} & \text { for }\left(\theta_{0}, \xi\right) \text { in region } A_{\mathrm{IV}}=A_{\mathrm{IV}}^{\prime} U A_{\mathrm{IV}}^{\prime \prime}\end{cases}$

Using Eqs. (12), (B2), and Fig. 10, the Fourier coefficient $C_{q}$ can be written as

$$
\begin{aligned}
C_{q}= & \int_{1-X}^{1} d \theta_{0} \int_{1-X}^{1} d \xi e^{2 \pi i(q-1) \xi}+\int_{1-X}^{1} d \theta_{0} \int_{0}^{1-X} d \xi e^{2 \pi i q \xi} \\
& +\int_{0}^{1-X} d \theta_{0} \int_{1-X-\theta_{0}}^{1-\theta_{0}} d \xi e^{2 \pi i(q-1) \xi} \\
& +\int_{0}^{1-X} d \theta_{0} \int_{0}^{1-X-\theta_{0}} d \xi e^{2 \pi i\left(q \xi+\theta_{0}\right)} \\
& +\int_{0}^{1-X} d \theta_{0} \int_{1-\theta_{0}}^{1} d \xi e^{2 \pi i\left(q \xi+\theta_{0}\right)}
\end{aligned}
$$

where the five integrals respectively correspond to the five regions, $A_{\mathrm{I}}, A_{\mathrm{II}}, A_{\mathrm{III}}, A_{\mathrm{IV}}^{\prime}$, and $A_{\mathrm{IV}}^{\prime \prime}$. Carrying out these integrations, we obtain Eq. (16).

[1] S. H. Strogatz, From Kuramoto to Crawford: Exploring the Onset of Synchronization in Populations of Coupled Oscillators, Physica D (Amsterdam) 143, 1 (2000).

[2] A. T. Winfree, The Geometry of Biological Time (SpringerVerlag, Berlin, 1980), Vol. 12.

[3] Y. Kuramoto, Chemical Oscillations, Waves, and Turbulence (Springer, Berlin, 1984).

[4] E. Ott, J. H. Platig, T. M. Antonsen, and M. Girvan, Echo Phenomena in Large Systems of Coupled Oscillators, Chaos 18, 037115 (2008).

[5] M. Herrera, T. M. Antonsen, E. Ott, and S. Fishman, Echoes and Revival Echoes in Systems of Anharmonically Confined Atoms, Phys. Rev. A 86, 023613 (2012).

[6] E. L. Hahn, Spin Echoes, Phys. Rev. 80, 580 (1950).

[7] G. Morigi, E. Solano, B.-G. Englert, and H. Walther, Measuring Irreversible Dynamics of a Quantum Harmonic Oscillator, Phys. Rev. A 65, 040102 (2002).

[8] T. Meunier, S. Gleyzes, P. Maioli, A. Auffeves, G. Nogues, M. Brune, J. M. Raimond, and S. Haroche, Rabi Oscillations Revival Induced by Time Reversal: A Test of Mesoscopic Quantum Coherence, Phys. Rev. Lett. 94, 010401 (2005).

[9] R. Gould, T. O’Neil, and J. Malmberg, Plasma Wave Echo, Phys. Rev. Lett. 19, 219 (1967).

[10] E. Ott, Relativistic Synchrotron Plasma Echo, J. Plasma Phys. 4, 471 (1970). 
[11] S. De Monte, F. d'Ovidio, S. Dan $\varnothing$, and P. G. Sørensen, Dynamical Quorum Sensing: Population Density Encoded in Cellular Dynamics, Proc. Natl. Acad. Sci. USA 104, 18377 (2007).

[12] J. Buck and E. Buck, Synchronous Fireflies, Sci. Am. 234, 74 (1976).

[13] J. Buck, Synchronous Rhythmic Flashing of Fireflies. II, Quart. Rev. Bio. 63, 265 (1988).

[14] I. Aihara, T. Mizumoto, T. Otsuka, H. Awano, K. Nagira, H. G. Okuno, and K. Aihara, Spatio-Temporal Dynamics in Collective Frog Choruses Examined by Mathematical Modeling and Field Observations, Sci. Rep. 4, 3891 (2014).

[15] E. A. Martens, S. Thutupalli, A. Fourriere, and O. Hallatschek, Chimera States in Mechanical Oscillator Networks, Proc. Natl. Acad. Sci. U.S.A. 110, 10563 (2013).

[16] A. N. Zaikin and A. M. Zhabotinsky, Concentration Wave Propagation in Two-Dimensional Liquid-Phase Self-Oscillating System, Nature (London) 225, 535 (1970).

[17] R. J. Field, E. Körös, and R. M. Noyes, Oscillations in Chemical Systems. II. Thorough Analysis of Temporal Oscillation in the Bromate-Cerium-Malonic Acid System, J. Am. Chem. Soc. 94, 8649 (1972).

[18] R. Toth and A. F. Taylor, Review of the tris (2,2'-Bipyridyl) Ruthenium Catalysed Belousov-Zhabotinsky Reaction, Prog. React. Kinet. Mech. 31, 59 (2006).

[19] A. F. Taylor, P. Kapetanopoulos, B. Whitaker, R. Toth, L. Bull, and M. Tinsley, Clusters and Switchers in Globally Coupled Photochemical Oscillators, Phys. Rev. Lett. 100, 214101 (2008).

[20] M. R. Tinsley, S. Nkomo, and K. Showalter, Chimera and Phase-Cluster States in Populations of Coupled Chemical Oscillators, Nat. Phys. 8, 662 (2012).

[21] S. Nkomo, M. R. Tinsley, and K. Showalter, Chimera States in Populations of Nonlocally Coupled Chemical Oscillators, Phys. Rev. Lett. 110, 244102 (2013).

[22] J. F. Totz, R. Snari, D. Yengi, M. R. Tinsley, H. Engel, and K. Showalter, Phase-Lag Synchronization in Networks of Coupled Chemical Oscillators, Phys. Rev. E 92, 022819 (2015).

[23] R. Snari, M. R. Tinsley, D. Wilson, S. Faramarzi, T. I. Netoff, J. Moehlis, and K. Showalter, Desynchronization of Stochastically Synchronized Chemical Oscillators, Chaos 25, 123116 (2015).

[24] S. Kadar, T. Amemiya, and K. Showalter, Reaction Mechanism for Light Sensitivity of the $\mathrm{Ru}(\mathrm{bpy})_{3}^{2+}$-Catalyzed Belousov-Zhabotinsky Reaction, J. Phys. Chem. A 101, 8200 (1997).
[25] A. F. Taylor, P. Kapetanopoulos, B. J. Whitaker, R. Toth, L. Bull, and M. R. Tinsley, Phase Clustering in Globally Coupled Photochemical Oscillators, Eur. Phys. J. Spec. Top. 165, 137 (2008).

[26] A. M. Zhabotinsky, F. Bucholtz, A. B. Kiyatkin, and I. R. Epstein, Oscillations and Waves in Metal-Ion-Catalyzed Bromate Oscillating Reactions in Highly Oxidized States, J. Phys. Chem. 97, 7578 (1993).

[27] Simulations were carried out with the two-variable ZBKE model [26] modified to describe the photosensitive discrete BZ oscillator system [19-21], where $\varepsilon_{1} d X_{j} / d t=\phi_{j}-X_{j}^{2}-X_{j}+\varepsilon_{2} \gamma u_{s s, j}^{2}+u_{s s, j}\left(1-Z_{j}\right)+$ $\left[\left(\mu-X_{j}\right) /\left(\mu-Z_{j}\right)\right]\left[q \alpha Z_{j} /\left(\varepsilon_{3}+1-Z_{j}\right)+\beta\right]$ and $d Z_{j} / d t=$ $2 \phi_{j}+u_{s s, j}\left(1-Z_{j}\right)-\alpha Z_{j} /\left(\varepsilon_{3}+1-Z_{j}\right) \quad$ describe the chemistry of oscillator $j$. The variables $X_{j}$ and $Z_{j}$ and the constant $q_{j}$ represent $\left[\mathrm{HBrO}_{2}\right],\left[\mathrm{Ru}(\mathrm{bpy})_{3}^{3+}\right]$, and the stoichiometric coefficient, respectively, associated with oscillator $j$. The photoexcitatory feedback associated with oscillator $j$ is $\phi_{j}$. The steady-state concentration of $\mathrm{HBrO}_{2}^{+}$, designated as $u_{s s}$, is determined for oscillator $j$ by the concentrations $x_{j}$ and $z_{j}$ according to $u_{s s, j}\left(x_{j}, z_{j}\right)=$ $\left(1 / 4 \gamma \varepsilon_{2}\right)\left(\sqrt{\left(16 \gamma x_{j} \varepsilon_{2}+z_{j}^{2}-2 z_{j}+1\right)}+z_{j}-1\right)$. Nondimensional model parameters are $\epsilon_{1}=0.11, \epsilon_{2}=$ $1.7 \times 10^{-5}, \quad \epsilon_{3}=1.6 \times 10^{-3}, \quad \gamma=1.2, \quad \alpha=0.10, \quad \beta=$ $1.7 \times 10^{-5}, \mu=2.4 \times 10^{-4}$, and a distribution in $q$ that gives a period of $42.5 \pm 2.4$.

[28] A noise term, $\eta_{i}$, is added to the differential equation for bromous acid for each oscillator, where $\eta_{i}$ has zero mean and is delta correlated in time with intensity $D$.

[29] H. Nakao, K. Arai, and Y. Kawamura, Noise-Induced Synchronization and Clustering in Ensembles of Uncoupled Limit-Cycle Oscillators, Phys. Rev. Lett. 98, 184101 (2007).

[30] T. M. O'Neil, Effect of Coulomb Collisions and Microturbulence on Plasma Wave Echo, Phys. Fluids 11, 2420 (1968).

[31] T. H. Jensen, J. H. Malmberg, and T. M. O’Neil, Measurement of Velocity Space Diffusion Using Plasma Wave Echo, Phys. Fluids 12, 1728 (1969).

[32] A. Y. Wong and D. R. Baker, Measurements of Diffusion in Velocity Space from Ion-Ion Collisions, Phys. Rev. 188, 326 (1969).

[33] C. C. Canavier and S. Achuthan, Pulse Coupled Oscillators and the Phase Resetting Curve, Math. Bosci. 226, 77 (2010). 